El Antoniano. 2018; 133: 27-43

Recepción: 15.02.2018

Aprobación: 18.10.2018

\title{
CALIDAD DEL AIRE EN LA CUENCA ATMOSFÉRICA DEL CUSCO
}

\author{
Juan Eduardo Gil Mora ${ }^{1}$. \\ ${ }^{1}$ Universidad Nacional de San Antonio Abad del Cusco. Docente Escuela de Posgrado. \\ Presidente del GESTA Aire-Cusco. mundoandino2005@yahoo.es
}

\section{RESUMEN}

La ciudad del Cusco se halla sobre los 3,340 m.s.n.m. es una ciudad de altura y está rodeada por colinas hacia el Norte, Este y Oeste y abierta hacia el Sur y Sur-Este; la dirección predominante de los vientos es de Sur-Este a Nor-Oeste, favoreciendo que los contaminantes producidos sean conducidos hacia el Centro Histórico; en el valle del Cusco se observa una inversión térmica de subsidencia entre los meses de mayo y septiembre agravando la concentración de polutantes. A través del Grupo de Estudio Técnico Ambiental del Aire -GESTA-aire, se ha evaluado las condiciones geomorfológicas determinándose la cuenca atmosférica, el inventario de emisiones de fuentes fijas y móviles y se ha estimado los volúmenes de contaminantes emitidos; el $84.65 \%$ de las emisiones corresponden a $\mathrm{PM}_{10}$ generadas por los 85,560 vehículos existentes en la ciudad; otra fuente importante son las fábricas de ladrillos y tejas. EI CO, representa el $9.92 \%$ de las emisiones totales siendo la principal fuente el parque automotor; otros contaminantes de importancia son el COV, NOx, SOx, generados por vehículos y ladrilleras, fábricas de tejas, pollerías, chicharronerías y viviendas. Se han estimado las emisiones totales para la ciudad del Cusco: PM $_{10}$ 151,553.2 Ton/año; CO 17,758.2 Ton/año; COV 6,308.2 Ton/año; NOx 2,684.5 Ton/año y SOx 699.6 Ton/año. La determinación de la calidad del aire, se realizó utilizando tubos pasivos y Hi-Vol; los métodos empleados para el cálculo son los desarrollados por la OMS, IPIECA y el Banco Mundial y el modelo MODEM para determinar las concentraciones totales.

Palabras claves: Cuenca atmosférica; inventario de emisiones; parque automotor; SOx; NOx.

\section{AIR QUALITY IN THE CUSCO ATMOSPHERIC BASIN}

\begin{abstract}
The city of Cusco is located at 3,340 m.a.s.I it is a city of height and it is surrounded by hills to the North, East and West and open to the South and South-East; the predominant direction of the winds is from South-East to North-West; this aspect implies that the pollutants produced by economic activities are directed towards the Historic Center; furthermore, in the Cusco valley a subsidence thermal inversion is observed between May and September, aggravating the concentration of pollutants. Through the Air Environmental Technical Study Group -GESTA- the geomorphological conditions have been evaluated, determining the atmospheric basin, the inventory of emissions from both fixed and mobile sources, and the volumes of pollutants emitted have been estimated; thus, $84.65 \%$ of the emissions correspond to $\mathrm{PM}_{10}$ generated by the 85,560 vehicles in the
\end{abstract}


city of Cusco; another important source is brick and tile factories. CO represents $9.92 \%$ of total emissions, the main source being the automotive fleet; other important pollutants are VOC, NOx, SOx, all generated by the automotive fleet and brick factories, tile factories, grilled chicken restaurants, pork rind restaurants and homes. Total emissions have been estimated for the city of Cusco: $\mathrm{PM}_{10}$ 151,553.2 Ton/year; CO 17,758.2 Ton/year; VOC 6,308.2 Ton/year; NOx 2,684.5 Ton/year and SOx 699.6 Ton/year. The evaluation was carried out using passive tubes and $\mathrm{Hi}$-Vol, the methods used for the calculation are those developed by the WHO, IPIECA and the World Bank and the MODEM model to determine total concentrations.

Keywords: Atmospheric basin; automotive fleet; emissions inventory; NOx; SOx.

\section{INTRODUCCIÓN}

En los últimos años la ciudad del Cusco ha tenido un crecimiento acelerado del parque automotor, generando problemas en la calidad del aire; de otro lado, se ha avanzado con la dación de normas sobre los Límites Máximos Permisibles y Estándares de Calidad Ambiental. No obstante, es necesario la decisión política de autoridades y sociedad civil para la puesta en marcha de las acciones de mitigación y prevención.

Las condiciones climáticas en la ciudad del Cusco ubicada en una plataforma andina, a lo largo de una cuenca de $31 \mathrm{~km}$ y a una altitud de 3,340 m.s.n.m. rodeada por colinas hacia el Norte y Oeste y abierta al Sur, condiciona la formación de un bolsón de aire seco, donde la dirección predominante de los vientos es de Sur a Norte (SENAMHI-PACC. 2011).

Las condiciones meteorológicas y fisiográficas, la alta densidad poblacional, el crecimiento acelerado del parque automotor, (se ha registrado en 1989, 11,806 vehículos en circulación y en el 2001, 33,316 unidades motorizadas, el crecimiento es del $282 \%$ en el lapso de 12 años, en el 2010, son 85,560 vehículos) han permitido que se agrave la contaminación atmosférica, especialmente en el centro histórico-monumental, lo cual tiene efectos en la salud y el bienestar de la población, y afecta la integridad del patrimonio monumental.

Se han planteado medidas para implementar el Plan a Limpiar el aire tales como la revisión obligatoria de emisiones vehiculares, la renovación completa de la flota del transporte público urbano y la restricción de la importación de vehículos usados a unidades de no más de tres años de antigüedad, la revisión y mejora de la planificación del transporte urbano y la introducción de alternativas para el transporte masivo, como metros o tranvías.

En cuanto a los niveles de contaminación encontrados en la evaluación de la calidad de aire realizada durante 1999 al 2004, en las calles y avenidas de la ciudad del Cusco por DIGESA, la UNSAAC, SENATI y la Municipalidad Provincial del Cusco a través del GESTA-Aire Cusco, se han registrado concentraciones de monóxido de carbono por encima de los límites permisibles $\left(10 \mathrm{ugCO} / \mathrm{m}^{3}\right)$, valor establecido por la OMS (1997) y todas las concentraciones registradas en partículas menores a 10 micrones $\left(\mathrm{PM}_{10}\right)$, excedieron los lineamientos de la OMS para 24 horas $\left(70 \mathrm{ug} / \mathrm{m}^{3}\right)$.

EI GESTA de Aire-Cusco definió la cuenca atmosférica de Cusco con el fin de enfocar los estudios de calidad de aire dentro de los límites geográficos planteados. Para la determinación de la cuenca atmosférica de Cusco se tomó en consideración la sub 
cuenca del río Huatanay que tiene un área de $502.18 \mathrm{~km}^{2}$, en cuyo seno se halla la ciudad del Cusco. Se ha desarrollado una zonificación de la cuenca, en la que se ha considerado tres anillos concéntricos (Figura 2):

Primer anillo o zona más impactada, ubicado alrededor del Centro Histórico del Cusco, lugar donde se concentra gran parte de la población, las actividades turísticas y comerciales, así como el parque automotor.

Segundo anillo, que abarca desde la parte alta de Picchu hasta Angostura en el distrito de San Jerónimo.

Tercer anillo, abarca desde la Microcuenca de Cachimayo en el distrito de San Sebastián hasta la Laguna de Huacarpay en el distrito de Lucre.

La cuenca atmosférica abarca las Provincias de Cusco y Quispicanchi, distritos de Cusco, Santiago, Wanchac, San Sebastian, Saylla, Oropesa y Lucre.

Figura 1: Delimitación de áreas de la Cuenca Atmosférica del Cusco

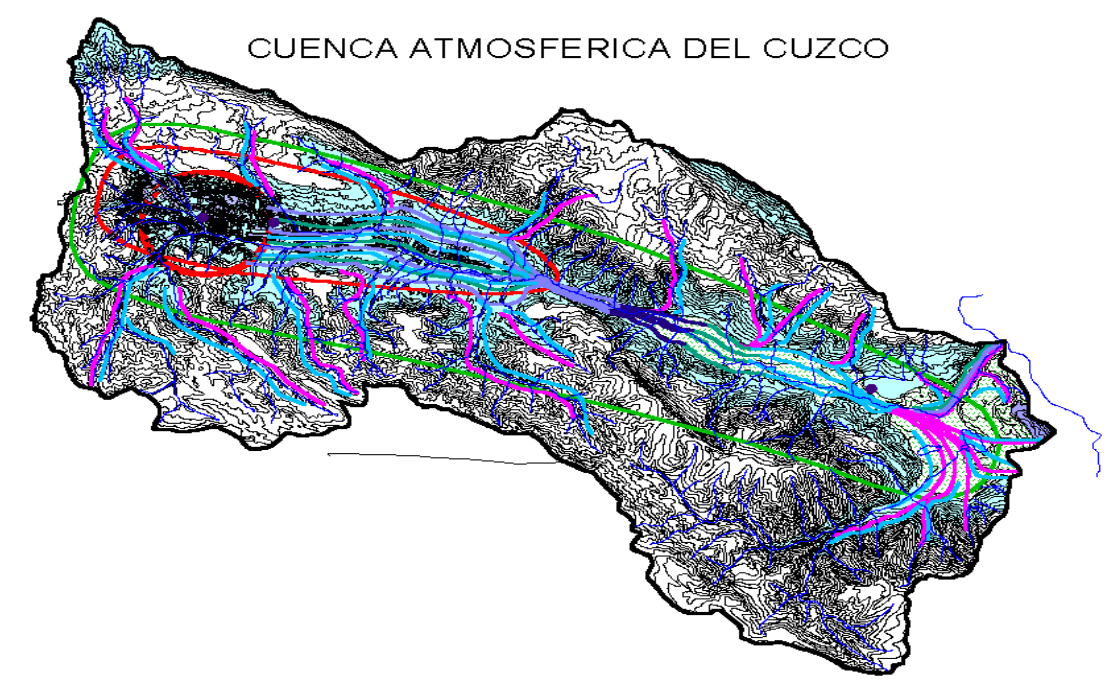

Fuente. GESTA-aire Cusco, elaborado por SENAMHI, 2009.

Figura 2. Zonificación de la cuenca atmosférica con identificación de tres anillos influenciados por la contaminación.

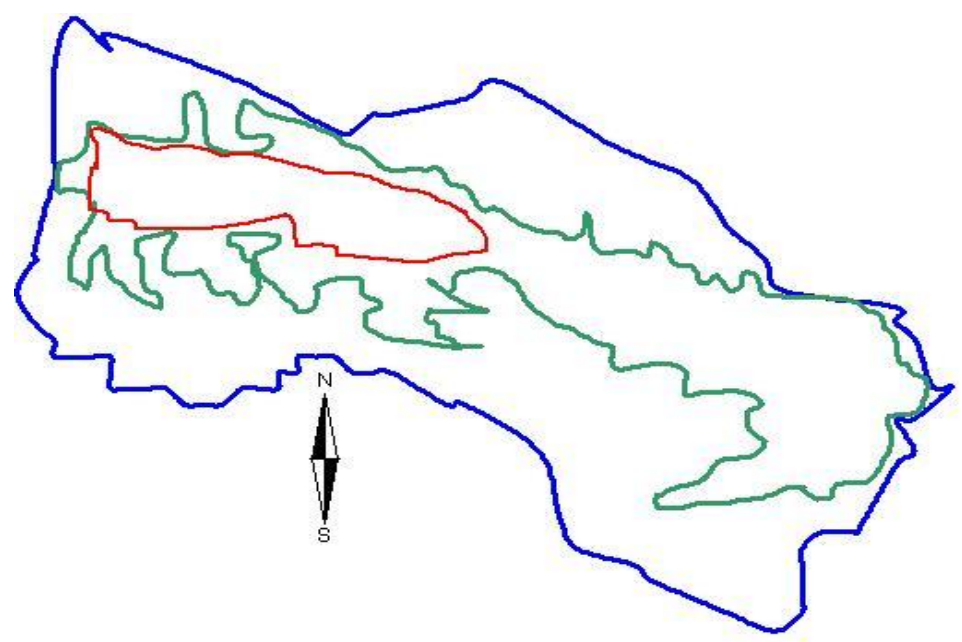


Fuente: Gesta Aire-Cusco. 2005

\section{Clima}

Cusco se halla bajo la influencia macro-climática de grandes masas de aire provenientes de la selva sur oriental, del Altiplano y de la Patagonia. Los vientos de la selva sur son masas de aire cargadas de humedad, que son impulsadas por los vientos alisios del oriente (SENAMHI-PACC, 2011).

Los vientos que llegan del Altiplano peruano-boliviano son fríos y secos, éstos ingresan por la zona sur oriental. Los vientos locales que se generan en los valles y llanuras del Cusco distribuyen calor y humedad a lo largo del día (SENAMHI-PACC, 2011).

De manera general se distinguen dos estaciones climáticas: la estación de lluvias, de noviembre a marzo y la estación de secas, de abril a octubre.

Figura 3. Temperatura y Precipitación Pluvial en Cusco.

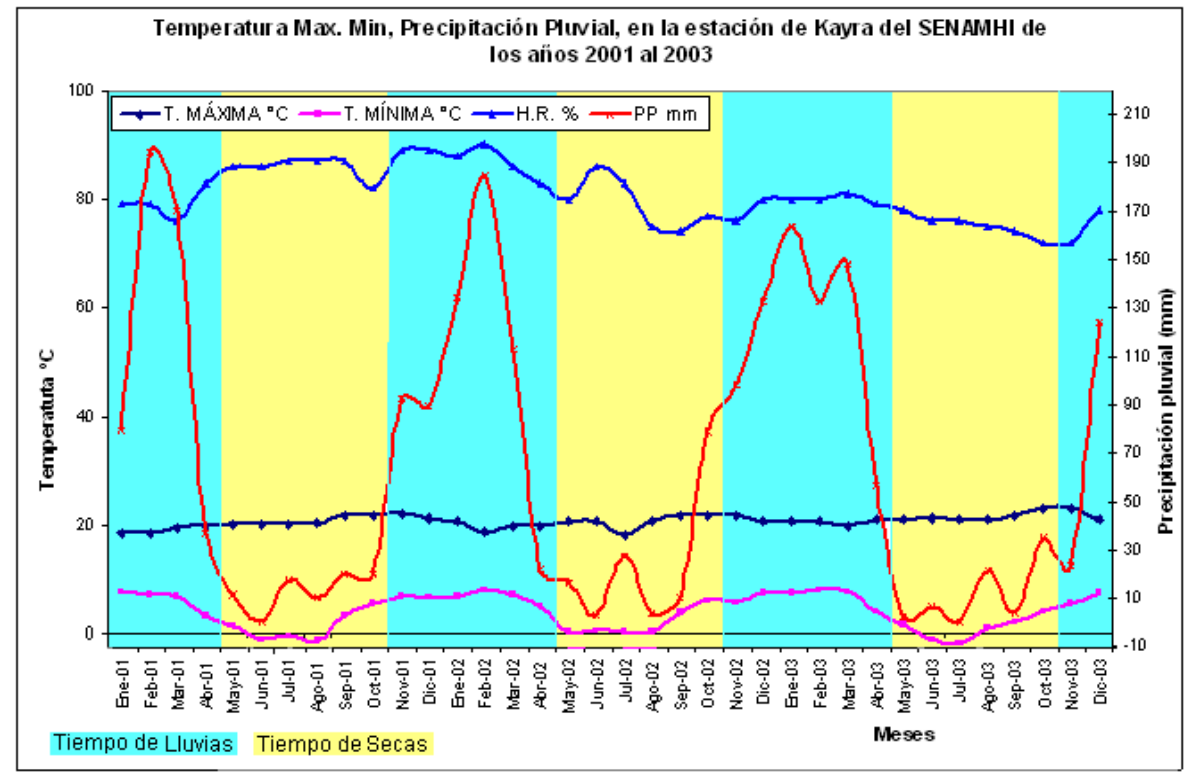

Fuente. Estación meteorológica de Kayra.

El grafico precedente muestra el comportamiento cíclico de la temperatura; la temperatura máxima, a través de los años se mantiene constante, mientras que la temperatura mínima tiene variaciones fuertes alcanzando sus niveles más bajos en los meses de mayo hasta agosto, en las primeras horas del día llega a valores bajo cero.

Figura 4. temperatura, humedad relativa y precipitación pluvial del año 1991 al 2001 


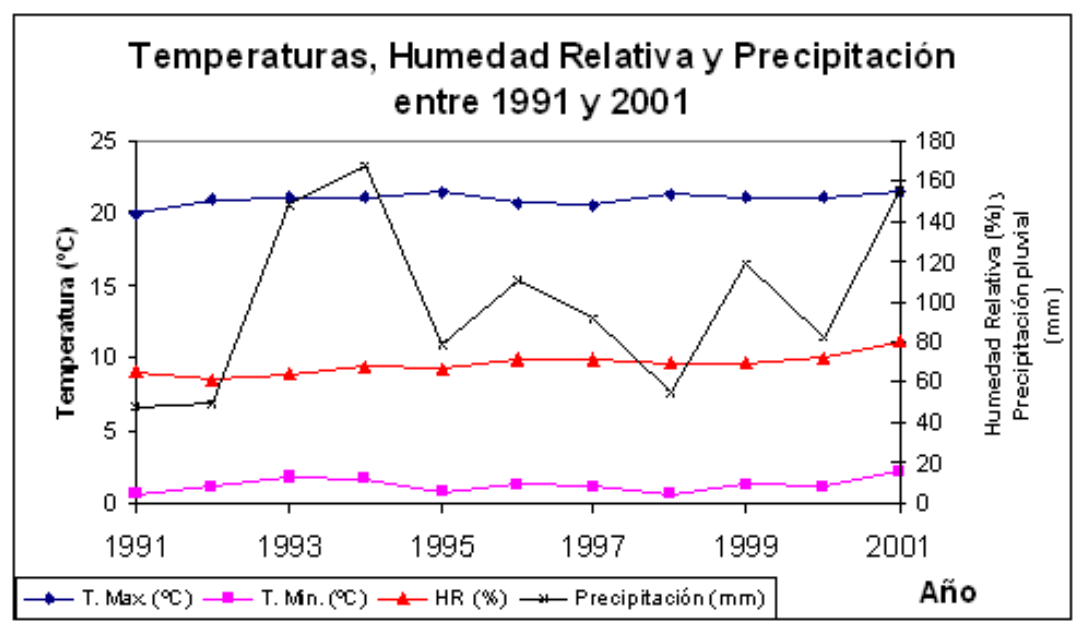

Fuente. Estación meteorológica de Kayra. 2005.

En la ciudad del Cusco, el mes más seco es junio, con $3 \mathrm{~mm}$ de precipitación y la mayor precipitación cae en enero, con un promedio de $154 \mathrm{~mm}$. El mes más caluroso del año es noviembre, con un promedio de $12.6^{\circ} \mathrm{C}$; julio tiene la temperatura promedio más baja del año con $8.9^{\circ} \mathrm{C}$.

En cumplimiento a las disposiciones nacionales; el 2001, se conformó el Grupo de Estudio Técnico Ambiental Aire del Cusco-GESTA aire cuyo objetivo es dar cumplimiento al Decreto Supremo N. 074-2001-PCM en la ciudad del Cusco; la actividad del GESTA, se puede resumir en lo siguiente:

1. Medidas políticas, al respecto, se han emitido:

- Ordenanza Municipal No. 005-99 y su reglamento para el control de gases tóxicos.

- Ordenanza Municipal No. 001-99, para el control de ruidos molestos.

2. Para el monitoreo y toma de muestras, se adquirieron los equipos:

- Analizador de gases marca HERMANN (Pieburg Instruments) Modelo MHC 218

- Opacímetro DO 285 petrolero marca HERMANN (Pierburg Instruments).

- Muestreador de Partículas HI-VOL para PM10, TSP y $\mathrm{PM}_{2.5}$

- Analizadores automáticos. Para gases criterio. Monitor de Ozono por absorción UV; Monitor de $\mathrm{SO}_{2}$ por fluorescencia; UV Monitor de $\mathrm{CO}$ por correlación IR; Monitor de $\mathrm{NO}, \mathrm{NO}_{2}$, NOx por quimioluminiscencia; Monitor de $\mathrm{HCT}\left(\mathrm{CH}_{4}\right.$ y $\left.\mathrm{HNM}\right)$ por ionización de flama.

3. En cuanto a normatividad:

- Reglamento Nacional para la aprobación de Estándares de Calidad Ambiental y Límites Máximos Permisibles, Decreto Supremo No. 0144-98-PCM.

- Reglamento de Estándares Nacionales de Calidad Ambiental del Aire, Decreto Supremo No. 074-2001-PCM.

- Programa Anual 2001 para Estándares de Calidad Ambiental y Límites Máximos permisibles. Resolución Presidencial No. 054-2001-CONAM/PCD.

- Límites Máximos Permisibles de emisiones de contaminantes para vehículos automotores que circulen en la red vial, Decreto Supremo No. 047-2001-MTC

4. Acciones realizadas al 2004:

- Monitoreo de análisis de la contaminación de aire en puntos críticos de la ciudad.

- Campañas de sensibilización para la ciudadanía y transportistas con relación a los peligros de la contaminación atmosférica. 
- Cursos de capacitación de contaminación de aire y control de gases de combustión.

- Ordenamiento del transporte masivo, establecimiento de paraderos fijos.

- Establecimiento de lunes perfectos de circulación vial.

\section{MATERIALES Y MÉTODOS}

\section{Determinación de la cuenca atmosférica}

Para su delimitación se utilizó la carta nacional en una escala de 1/100000 y los planos cartográficos proporcionados por integrantes del GESTA, especialmente por el PER-IMA y PER-Plan MERISS; el valle del Cusco, donde se sitúa la cuenca atmosférica materia de estudio, tiene aproximadamente $30 \mathrm{~km}$ de largo, empieza en las cumbres de los montes Senca y Ulluchani, a 4,514 y $4,437 \mathrm{msnm}$, respectivamente, y termina en la confluencia del río Huatanay con el río Vilcanota. La cuenca se divide en dos depresiones topográficas, la del Cusco y la de Oropesa. La primera, más ancha e importante, tiene $13.5 \mathrm{~km}$ de largo y se despliega entre el inicio del valle y el sector Angostura, donde se estrecha para formar un callejón de $250 \mathrm{~m}$ de ancho y $2 \mathrm{~km}$ de largo y desembocar en la segunda; esta tiene $15.5 \mathrm{~km}$ de largo y comprende desde el citado sector hasta la laguna Lucre-Huacarpay, culminando en el sitio denominado Rumicolca. Otro aspecto considerado para la delimitación de la cuenca y su zonificación, es la mayor actividad productiva, comercial y de servicio, que comprende hasta el sector denominado Angostura, ubicado en el distrito de San Jerónimo, denominándose a este sector como Zona de Atención Prioritaria (zona demarcada de rojo en las Figura 1 y 2).

\section{Estimación de emisiones}

La información para el inventario de las fuentes de emisión, se obtuvo a través de encuestas y extrapolación de los resultados. Los métodos empleados para el cálculo son los desarrollados por la OMS (2009), IPIECA (International Petroleum Industry Environmental Conservation Association. 2019)) y el Banco Mundial (2000).

Las estimaciones de las emisiones que son vertidas a la atmósfera mediante chimeneas o ductos de ventilación han sido calculadas mediante métodos directos e indirectos; ambos, requieren trabajos de campo para recolectar información; esto es, el muestreo en la fuente de emisión; éste es una prueba que se realiza directamente en la fuente de emisión, es decir, en la chimenea o ducto por donde se dirigen los gases contaminantes hacia el aire ambiente (MINAMBIENTE, 2017). Esta prueba posibilita obtener la concentración, es decir, la tasa de la masa del contaminante y el volumen de aire que lo transporta (EPA, 1997); las estaciones de muestreo han sido definidas por especialistas de DIGESA basados en criterios de dirección predominante de vientos y presencia de fuentes emisoras de contaminantes.

De conformidad a EPA (2019) y a la Asociación de Gobernadores del Oeste Denver Colorado y el Comité Asesor Binacional. Radian International (1997), un inventario de emisiones atmosféricas constituye un conjunto de datos que caracterizan y consolidan, mediante sumatoria, las emisiones de contaminantes atmosféricos, de acuerdo con el tipo de fuente y el tipo y cantidad de contaminantes emitidos, en un área geográfica y en un intervalo de tiempo determinados, para la cuenca atmosférica del Cusco, se aplicó la metodología propuesta por la EPA, para ello se empleó la ecuación:

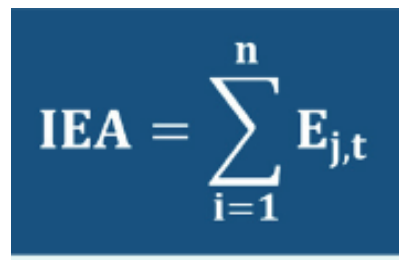


Donde:

IEA: inventario de emisiones atmosféricas para la sustancia o mezcla de sustancias (j) en el periodo de tiempo $(\mathrm{t})$.

Ej,t: emisión atmosférica de la sustancia o mezcla de sustancias (j), generada por la actividad (i) en el periodo de tiempo (t).

$\mathrm{n}$ : número total de actividades a inventariar.

Además de la ecuación precedente, en la mayoría de los casos no es posible contar con información de las emisiones medidas de forma directa durante el periodo de tiempo de evaluación en cada una de las fuentes a inventariar, el enfoque más común para estimar las emisiones en un inventario $(E j, t)$, consiste en combinar información de los procesos de cada actividad, con información de las emisiones asociadas a los procesos de dicha actividad (SEMARNAT, 2012); por lo tanto, se empleó la siguiente ecuación:

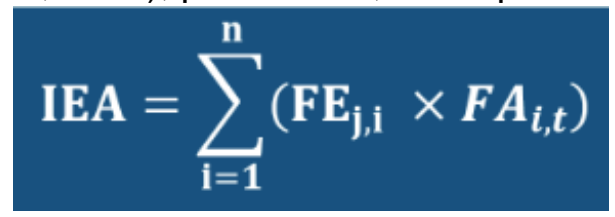

Donde:

FEj,i: factor de emisión de la sustancia o mezcla de sustancias ( j) para la actividad (i).

FAi,t: factor de actividad de la actividad (i) durante el tiempo (t).

El inventario de emisiones de fuentes fijas en la cuenca atmosférica del Cusco, fue realizado aplicando la metodología de Evaluación de Fuentes de Contaminación del Aire (Técnicas para el Inventario Rápido de la Contaminación Ambiental de Alexander P. Economopoulos, traducido y publicado por el Centro Panamericano de Ingeniería Sanitaria y Ciencias del Ambiente-CEPIS de la Organización Mundial de la Salud, a la que denominamos Metodología OMS, 1993).

\section{Emisiones vehiculares}

Para las emisiones vehiculares, una vez obtenida la información de campo, utilizando el opacímetro y el analizador de gases, la metodología para estimar las concentraciones en la cuenca atmosférica, fue utilizando el modelo de emisiones Vehiculares-MODEM; este modelo corresponde a una metodología y a un software especializado que permite calcular las emisiones de contaminantes atmosféricos producidas por la actividad vehicular terrestre en zonas urbanas (Osses Alvarado, M. 2000); es un programa que utiliza corridas del modelo Estraus y permite obtener emisiones de contaminantes específicos asociados a distintas categorías vehiculares, generando resultados en base horaria o anual.

El MODEM, incluye los siguientes contaminantes: $\mathrm{CO}$, VOC, $\mathrm{NOx}$ (NO2 y NO), $\mathrm{CO} 2, \mathrm{CH} 4$, $\mathrm{N} 2 \mathrm{O}, \mathrm{SO}, \mathrm{NH} 3, \mathrm{PM}_{10}, \mathrm{PM}_{2.5}$, PST.

Según Bravo Ordoñez A. et Al. (2000), la metodología de cálculo implementada en MODEM asume que las emisiones provienen de tres fuentes: las derivadas del motor cuando éste se encuentra en condiciones de operación estables (emisiones en caliente), 
aquellas provenientes del motor cuando éste se encuentra frío (emisiones por partidas en frío) y aquellas denominadas evaporativas (emisiones de hidrocarburos evaporados). Las emisiones totales serán, en consecuencia, la suma de estos tres tipos de emisiones, es decir:

$$
E_{\text {total }}=E_{\text {caliente }}+E_{\text {partidas en frío }}+E_{\text {evaporativas }}
$$

Donde:

Etotal: Emisiones totales del contaminante considerado [gramos]

Ecaliente: Emisiones en caliente, fase estabilizada del motor [gramos]

Epartidas en frío: Emisiones por partidas en frío [gramos]

Eevaporativas: Emisiones por evaporación [gramos]

\section{Tipos de Fuentes Emisoras}

Con la finalidad de identificar las fuentes de emisión, éstas han sido agrupadas como fuentes puntuales, a toda instalación establecida en un lugar que desarrollan operaciones o procesos industriales o actividades que puedan generar emisiones contaminantes significativas a la atmósfera (INECC, 2002), por ejemplo, se puede citar las fundiciones primarias, pollerías, fábrica de tejas y ladrillos y toda actividad económica que tiene un emisor fijo. Fuentes de Área, aquellas que no poseen un foco emisor como el sector doméstico, comercial y de servicios, como las panaderías, talleres de carpintería, grifos y otros.

Para la determinación del Universo de fuentes emisoras, se utilizó la información proporcionada por la Municipalidad provincial del Cusco y la Cámara de Comercio del Cusco.

\section{Calidad del aire ambiente}

Para la determinación de la calidad del aire ambiente, se utilizaron tubos pasivos y muestreadores activos como el Hi-vol y un equipo analizador automático (Ver Figuras 5, 6 y 7).

Figura 5. Tubos pasivos listos para su operación, se instalaron 21 estaciones de muestreo en la cuenca atmosférica del Cusco.

Figura 6. Muestreador de y $\mathrm{PM}_{2.5}$.

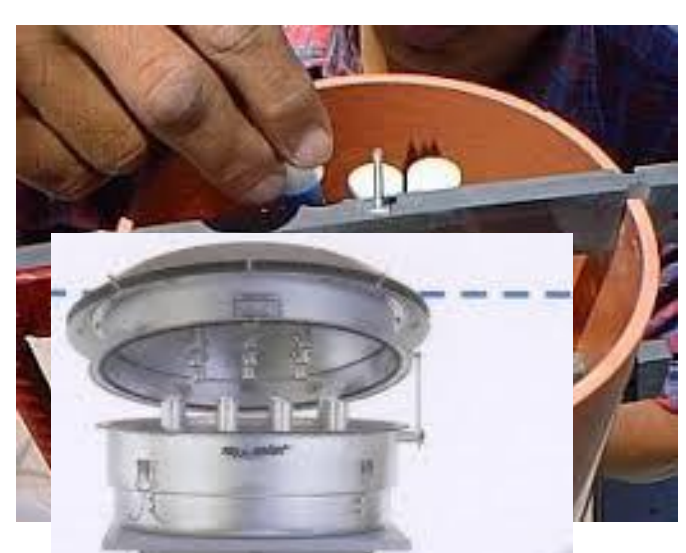

Partículas HI-VOL para $\mathrm{PM}_{10}$, TSP 
Figura 7. Equipo analizador automático.

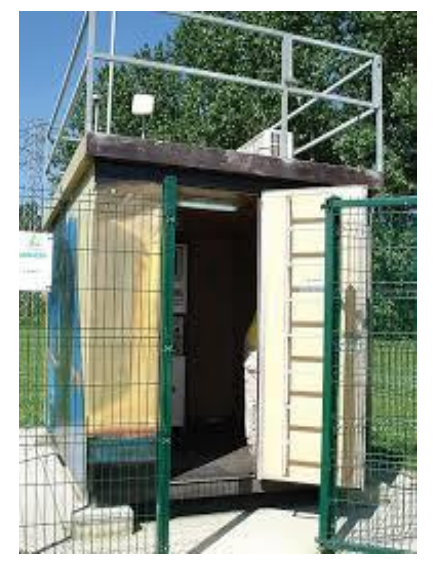

Para el estudio de la calidad del aire ambiente, se seleccionaron el dióxido de nitrógeno $\left(\mathrm{NO}_{2}\right)$ y dióxido de azufre $\left(\mathrm{SO}_{2}\right)$ como contaminantes a medir. $\mathrm{El} \mathrm{NO}$ representa la contaminación causada por el transporte rodante (privado, público y taxis). $\mathrm{El} \mathrm{SO}_{2}$ normalmente proviene del uso de los combustibles en el parque automotor y actividades industriales; usualmente el contenido de azufre en los combustibles tipo diesel es alto (Anuario Estadístico de Hidrocarburos 2000).

Las estaciones de muestreo se eligieron en función a las actividades económicas, la congestión vehicular, el tipo de calles. Se seleccionaron 15 estaciones, para la medición de $\mathrm{NO}_{2}$ y $\mathrm{SO}_{2}$, y material particulado localizados en zonas de alto tráfico, zonas comerciales y zonas residenciales, la selección de estas estaciones se hizo buscando abarcar la mayor parte de la cuenca atmosférica.

Los tubos pasivos fueron colocados en 21 estaciones referenciales a una altura de seis metros y, fueron cambiados por unos nuevos cada mes de conformidad a la metodología recomendada para una apropiada medición (Comisión Nacional del Medio Ambiente, 2009; EPA, 1999; Robinson, E.et Al, 1968). 


\section{RESULTADOS Y DISCUSIÓN}

De conformidad al inventario vehicular, el número de unidades vehiculares alcanzan a 85,560 , predominando los automóviles sean privados y los de servicio público (taxis), ambos constituyen el $71.4 \%$ del total de unidades para la ciudad del Cusco; referente a las unidades de servicio de transporte público (buses y combis), estas alcanzan un porcentaje de $13.7 \%$.

Cuadro 1. Parque automotor en la cuenca atmosférica del Cusco.

\begin{tabular}{|c|c|c|}
\hline $\begin{array}{ll}\text { Tipo } & \text { de } \\
\text { vehículo } & \\
\end{array}$ & $\begin{array}{ll}\text { Número } & \text { de } \\
\text { unidades }\end{array}$ & Porcentaje \\
\hline Automóvil & 33,693 & 39.38 \\
\hline Bus & 9,040 & 10.56 \\
\hline Camión & 3,415 & 04.00 \\
\hline Camioneta & 9,293 & 10.86 \\
\hline Combi & 2,706 & 03.16 \\
\hline Taxi & 27,413 & 32.04 \\
\hline TOTAL & 85,560 & 100.00 \\
\hline
\end{tabular}

Fuente: Inventario de emisiones Cusco. GESTA. 2004

Contrastando la información del Cuadro 1 con resultados de inventarios vehiculares en otras ciudades; es el caso señalado por la Comisión Nacional del Ambiente (2009) que indica que la principal fuente de emisión son los vehículos de servicio público (buses); igualmente, Giraldo et Al (2009) menciona que los buses por contar un mayor número de unidades constituyen una principal fuente de emisión; en Cusco, en razón a que buses y combis constituyen el $13.72 \%$ del total de unidades vehiculares existentes en la cuenca atmosférica, sus emisiones deben ser consideradas dentro del total de las fuentes de emisión.

De otro lado, el inventario de emisiones arroja un total de 2,095 fuentes fijas de contaminación, tal como lo señala el Cuadro 2; cabe resaltar que las fuentes de emisión más importantes están conformadas por panaderías, ladrilleras, carpinterías y aserraderos, uso de solventes y pinturas, restaurantes, pollerías y pizzerías, y las imprentas.

Cuadro $\mathrm{N}^{\circ}$ 2. Principales fuentes de contaminación atmosférica en Cusco

\begin{tabular}{|c|c|c|c|c|}
\hline No & Estrato & Descripción & Nombre Común & $\begin{array}{l}\text { Total Fuentes en la } \\
\text { Cuenca Atmosférica }\end{array}$ \\
\hline 1 & $\begin{array}{l}\text { Actividades de la industria } \\
\text { alimentaria }\end{array}$ & Industria cervecera & - & 01 \\
\hline 2 & \multirow{3}{*}{$\begin{array}{l}\text { Actividades comerciales y } \\
\text { de servicios que realizan } \\
\text { combustión }\end{array}$} & $\begin{array}{l}\text { Restaurantes, cafés y otros establecimientos que } \\
\text { expenden comidas y bebidas }\end{array}$ & Pollerías / Pizzerías & 106 \\
\hline 3 & & Fabricación de productos de panadería & Panaderías & 375 \\
\hline 4 & & Suministro de vapor y agua caliente & Hoteles / Hospitales & 120 \\
\hline 5 & $\begin{array}{l}\text { Actividades productivas de } \\
\text { molinería }\end{array}$ & Molienda de granos & Molinos & 88 \\
\hline 6 & $\begin{array}{l}\text { Actividades artesanales } \\
\text { que realizan combustión }\end{array}$ & $\begin{array}{l}\text { Fabricación de productos cerámicos para la } \\
\text { construcción }\end{array}$ & $\begin{array}{l}\text { Ladrilleras } \\
\text { artesanales }\end{array}$ & 300 \\
\hline 7 & \multirow[b]{2}{*}{$\begin{array}{l}\text { Actividades de la industria } \\
\text { no metálica }\end{array}$} & Fabricación de artículos de hormigón & Bloqueteras & 74 \\
\hline 8 & & Transporte de materiales de construcción & $\begin{array}{l}\text { Transporte de } \\
\text { materiales de } \\
\text { construcción }\end{array}$ & 40 \\
\hline 9 & $\begin{array}{l}\text { Actividades de } \\
\text { transformación de madera }\end{array}$ & Carpinterías / Aserraderos & $\begin{array}{l}\text { Carpinterías / } \\
\text { Aserraderos }\end{array}$ & 355 \\
\hline 10 & $\begin{array}{l}\text { Pérdidas evaporativas por } \\
\text { expendio de combustible }\end{array}$ & $\begin{array}{l}\text { Venta al por menor de combustibles para } \\
\text { automotores }\end{array}$ & Grifos & 40 \\
\hline 11 & \multirow{2}{*}{$\begin{array}{l}\text { Evaporación de solventes } \\
\text { por fuentes de área }\end{array}$} & Uso de pinturas y solventes & $\begin{array}{l}\text { Uso de pinturas y } \\
\text { solventes }\end{array}$ & 351 \\
\hline 12 & & Actividades de impresión & Imprentas & 245 \\
\hline \multicolumn{4}{|c|}{ Total Fuentes Puntuales } & 01 \\
\hline \multirow{2}{*}{\multicolumn{4}{|c|}{\begin{tabular}{|l|} 
Total Fuentes de Area \\
Total Fuentes Fijas
\end{tabular}}} & 2094 \\
\hline & & & & 2095 \\
\hline
\end{tabular}


Fuente. Inventario de emisiones. Gesta Aire Cusco. 2001

Más del $43 \%$ de las fuentes fijas existentes en la cuenca atmosférica está integrado por restaurantes, pollerías, pizzerías, panaderías, hospedajes y ladrilleras; esto implica que estas fuentes son las que más contaminación generan en la cuenca Cusco. En cambio, las fuentes de área sin chimenea (carpinterías y aserraderos, grifos uso de pinturas y solventes e imprentas), constituyen el $47 \%$ del total de las fuentes; otro rubro importante.

Comparando los resultados de la Tabla 2 con otras investigaciones, señalamos que, según el BID (2013), EPA (1997) y Radian (1996), las fuentes fijas con un dispositivo puntual de emisión, son las que mayores concentraciones de contaminantes generan en una cuenca atmosférica; el inventario de fuentes en Cusco, ratifica este hecho. De Nevers (1998), recomienda que, para la planificación en el control de emisiones, es imprescindible considerar las fuentes de área; en efecto, al inventariar este tipo de fuentes para Cusco, se reporta que son 1,153 establecimientos a ser considerados.

DIGESA (2005), concluye que las fuentes de área son difíciles de medir y cuantificar sus emisiones, hecho que ha sido ratificado por la presente investigación.

En Cusco, el parque automotor constituye una de las principales fuentes de emisión de contaminantes, el inventario y las evaluaciones de contaminantes arroja lo siguiente:

Cuadro 3. Niveles de Emisiones generadas por el Parque Automotor del Cusco (Ton/año)

\begin{tabular}{|c|c|c|c|c|c|c|c|}
\hline Categoría & $\mathrm{SO}_{\mathrm{x}}$ & $\mathrm{NO}_{\mathrm{x}}$ & $\mathrm{CO}$ & $\mathrm{Pb}$ & cov & $\begin{array}{l}\mathrm{PM}_{10} \\
\text { Combustión }\end{array}$ & $\begin{array}{l}\mathrm{PM}_{10} \\
\text { Fugitivo }\end{array}$ \\
\hline Automóvil particular & 1.7 & 11.8 & 133.9 & 0.6 & 19.1 & 0.5 & 1027.4 \\
\hline Automóvil taxi & 51.3 & 351.1 & 3560.2 & 17.5 & 526.5 & 17.3 & 30850.1 \\
\hline Bus $<25$ & 233.6 & 917.8 & 466.7 & 0.0 & 202.2 & 70.0 & 27995.2 \\
\hline Bus $>25$ & 173.8 & 685.0 & 429.6 & 0.3 & 158.1 & 52.3 & 29442.4 \\
\hline Camion & 46.7 & 183.4 & 93.2 & 0.0 & 40.4 & 14.0 & 8955.1 \\
\hline Camioneta Panel & 1.1 & 8.6 & 75.1 & 0.4 & 9.6 & 0.3 & 1329.9 \\
\hline Camioneta Pickup & 13.5 & 27.6 & 133.9 & 0.8 & 22.5 & 3.3 & 6607.3 \\
\hline Combi comercial & 1.3 & 1.1 & 1.6 & 0.0 & 0.4 & 0.3 & 415.0 \\
\hline Combi público & 54.0 & 61.9 & 280.8 & 1.0 & 35.5 & 13.2 & 20520.9 \\
\hline $\begin{array}{ll}\text { Station } & \text { Wagon } \\
\text { particular } & \end{array}$ & 1.0 & 7.5 & 66.4 & 0.4 & 10.8 & 0.3 & 848.4 \\
\hline Station Wagon taxi & 27.6 & 181.6 & 1607.5 & 9.3 & 239.7 & 8.0 & 20818.2 \\
\hline TOTAL & 605.6 & 2437.4 & 6848.9 & 30.3 & 1264.8 & 179.5 & 1488009.9 \\
\hline
\end{tabular}

Fuente: DIGESA-Gesta Aire Cusco. 2004

El cuadro 3 identifica al sector del parque automotor que tiene las mayores emisiones, la categoría automóvil, camión y camionetas no tienen mayores aportes, sin que esto signifique que no contribuyen a la contaminación, es el sector transporte público (Combis y buses y otros servicios como el de turismo y taxis), los que generan los mayores aportes de emisiones, esta distribución de emisiones se aprecia también en la Figura 8, donde se observa que el mayor contaminante emitido es el $\mathrm{CO}$, seguido del $\mathrm{NO}_{\mathrm{x}}, \mathrm{COV}$ y $\mathrm{SO}_{\mathrm{x}}$.

Los vehículos que utilizan gasolina como combustible (tienen altas emisiones de $\mathrm{CO}$, $\mathrm{COV}, \mathrm{Pb}$ y $\mathrm{NO}_{\mathrm{x}}$ ), en contraposición, los vehículos que utilizan diesel como combustible tienen altas emisiones de $\mathrm{SO}_{x}$ por contener el diesel altas concentraciones de azufre, $\mathrm{NO}_{x}$ por las elevadas temperaturas alcanzadas en este tipo de motores combinando el 
nitrógeno del aire; este combustible genera emisiones importantes de $\mathrm{PM}_{10}$ respecto de los gasolineros.

Figura 8. Emisión de contaminantes - Parque automotor del Cusco

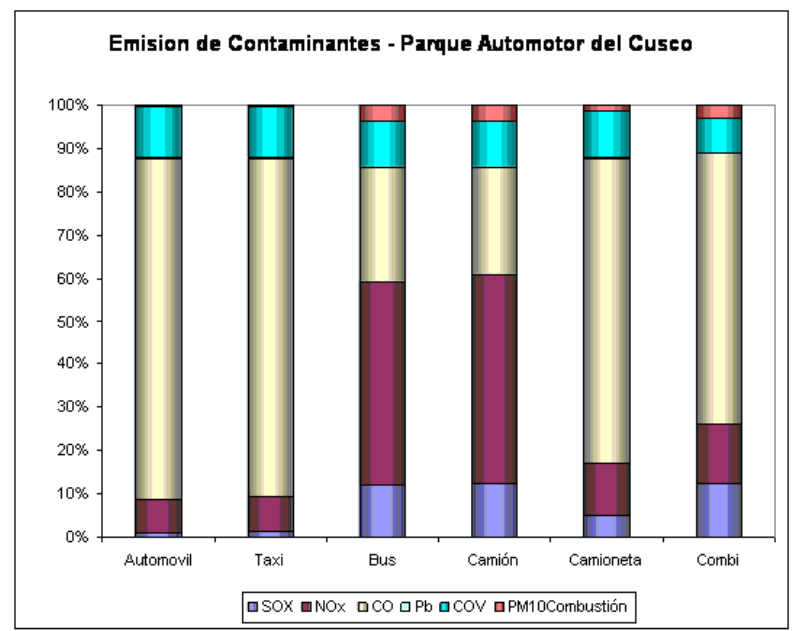

Fuente. GESTA-aire Cusco. 2004.

El transporte público en Cusco (taxi, bus y combi), es el que genera más del $89 \%$ de emisiones de SOx; 90.1\% de NOx; 92.6\% de CO; 91.9\% de COV. Esto se debe a las horas de circulación, la edad del vehículo y el tipo de combustible que emplean. Al comparar estos resultados con otros estudios, se tiene que Blazer (2000), indica que del total de emisiones en la cuenca Lima-Callao, el 65\% corresponde a unidades vehiculares de servicio público; nuestros estudios en los parámetros anotados superan el $90 \%$. D'Angiola et AI (2010) y el BID (2013) concluyen que el tipo de combustible utilizado en el tráfico vehicular contribuye a la emisión de SOx, en efecto este hecho es corroborado por nuestros resultados. La OMS (1997), concluye que los NOx son generados mayormente por vehículos movidos por gasolina, aspecto que también se observa en los vehículos en Cusco.

Figura 9. Emisiones vehiculares, según categoría.

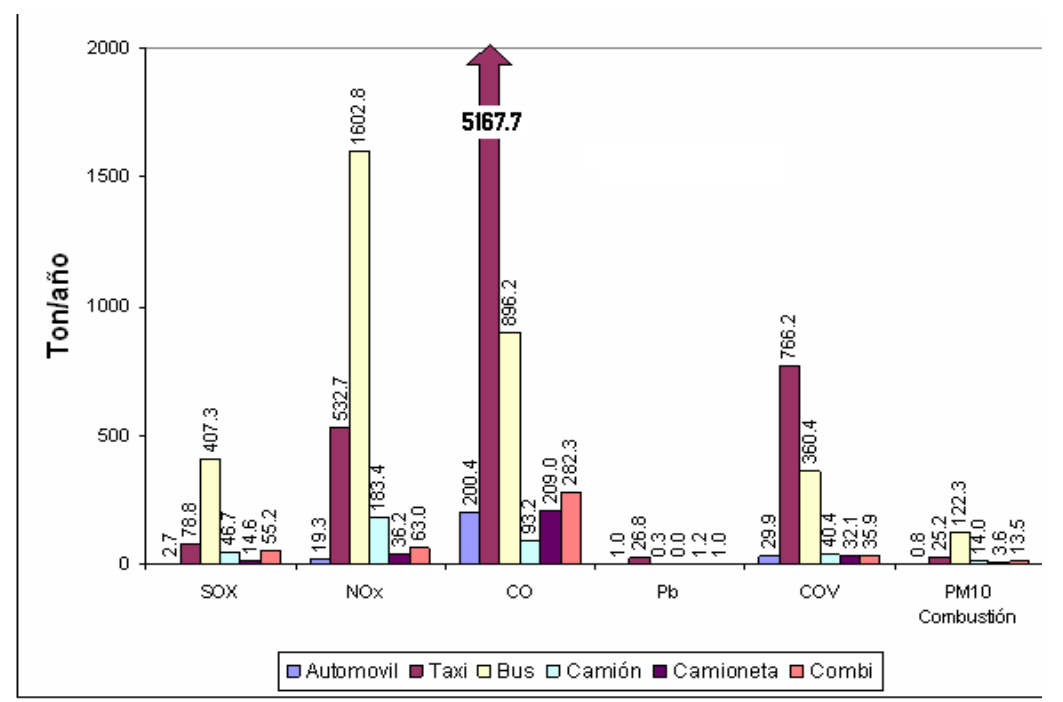

Fuente. Cuadro 3. GESTA-aire Cusco. 2004. 
Cuadro 4. Niveles de Emisiones, según rubro de actividad para la Cuenca Atmosférica del Cusco (Ton/año).

\begin{tabular}{|l|l|l|l|l|l|l|}
\hline Actividad & $\mathbf{P M}_{10}$ & $\mathbf{S O}_{\mathbf{x}}$ & $\mathbf{N O}_{\mathbf{x}}$ & $\mathbf{C O}$ & $\mathbf{C O V}$ & $\mathbf{P b}$ \\
\hline $\begin{array}{l}\text { Uso solventes y pintura (talleres } \\
\text { mecánicos y vivienda) }\end{array}$ & 0.0 & 0.00 & 0.00 & 0.00 & 282.83 & 0.00 \\
\hline $\begin{array}{l}\text { Fabricación de alimentos } \\
\text { (molineras) }\end{array}$ & 664.2 & 0.00 & 0.00 & 0.00 & 0.00 & 0.00 \\
\hline Aserraderos y carpinterías & 3.8 & 0.00 & 0.00 & 0.00 & 4.57 & 0.00 \\
\hline \begin{tabular}{l} 
Imprentas \\
\hline Ladrilleras y tejerías
\end{tabular} & 0.0 & 0.00 & 0.00 & 0.00 & 1.71 & 0.00 \\
\hline $\begin{array}{l}\text { Uso calderos y calentadores } \\
\text { (hoteles y hospitales) }\end{array}$ & 1067.3 & 7.96 & 55.75 & 5575.44 & 1831.93 & 0.00 \\
\hline Pollerías & 0.7 & 26.63 & 6.45 & 3.77 & 1.41 & 0.00 \\
\hline Panaderías & 38.3 & 0.78 & 2.08 & 132.27 & 47.62 & 0.00 \\
\hline Venta de combustibles (grifos) & 134.3 & 5.41 & 13.37 & 1252.94 & 526.68 & 0.00 \\
\hline Transporte vehicular & 0.0 & 0.00 & 0.00 & 0.00 & 135.67 & 0.00 \\
\hline Transporte aéreo & 148989.4 & 605.33 & 2437.47 & 6848.87 & 1264.94 & 30.26 \\
\hline Lavanderías & 5.0 & 7.60 & 72.00 & 0.00 & 103.40 & 0.00 \\
\hline Viviendas & 0.0 & 0.00 & 0.00 & 0.00 & 112.80 & 0.00 \\
\hline TOTAL & 650.2 & 45.89 & 97.41 & 3944.96 & 1994.69 & 0.00 \\
\hline
\end{tabular}

Fuente: DIGESA-Gesta Aire Cusco. 2004

El $84.65 \%(151,553.2$ ton/año) de las emisiones generadas en la cuenca atmosférica del Cusco, corresponde a $\mathrm{PM}_{10}$, la mayor carga de $\mathrm{PM}_{10}$ corresponde al Transporte Vehicular; otra actividad que aporta $\mathrm{PM}_{10}$ en forma significativa son las fábricas de ladrillos y tejas. Las fuentes de emisión de CO son: ladrilleras, panaderías, transporte vehicular y las viviendas, que en conjunto aportan más del $99 \%$ de este contaminante; dos fuentes (ladrilleras y el transporte vehicular) emiten el $70 \%$ de CO.

Cuadro 5. Niveles de Emisiones para la Cuenca Atmosférica del Cusco (Ton/año) 


\begin{tabular}{|l|l|l|l|l|l|l|}
\hline Descripción & $\mathbf{P M}_{10}$ & $\mathbf{S O}_{\mathbf{x}}$ & $\mathbf{N O}_{\mathbf{x}}$ & $\mathbf{C O}$ & $\mathbf{C O V}$ & $\mathbf{P b}$ \\
\hline Ladrilleras y tejerías & 1067.3 & 8.0 & 55.8 & 5575.4 & 1831.9 & 0.0 \\
\hline Panadería & 134.3 & 5.4 & 13.4 & 1252.9 & 526.7 & 0.0 \\
\hline Transporte vehicular & 148989.4 & 605.3 & 2437.5 & 6848.9 & 1264.9 & 30.3 \\
\hline Viviendas & 650.2 & 45.9 & 97.4 & 3945.0 & 1994.7 & 0.0 \\
\hline Otros & 712.1 & 35.0 & 80.5 & 136.0 & 690.0 & 0.0 \\
\hline TOTAL & $\mathbf{1 5 1 5 5 3 . 2}$ & $\mathbf{6 9 9 . 6}$ & $\mathbf{2 6 8 4 . 5}$ & $\mathbf{1 7 7 5 8 . 2}$ & $\mathbf{6 3 0 8 . 2}$ & $\mathbf{3 0 . 3}$ \\
\hline
\end{tabular}

Fuente: DIGESA-Gesta Aire Cusco. 2004

La generación de CO, 17,758.2 ton/año representa el 9.92\% de las emisiones totales, el transporte vehicular $(6,848.9$ ton/año) representa el $38.6 \%$ de estas emisiones, las ladrilleras y tejerías emiten el $31.4 \%$ de las emisiones y las viviendas el $22.2 \%$.

El COV representa el 3.52\% (6,308.2 ton/año) de las emisiones totales, las viviendas, ladrilleras y tejerías aportan el 31.6 y $29.0 \%$ de estas emisiones respectivamente, seguido del transporte vehicular con $20.1 \%$.

La generación de $\mathrm{NO}_{x}$, que corresponde al $1.50 \%$ (2,684.5 ton/año) de las emisiones totales, está producido por el transporte vehicular (90.8\%) que por efectos de combustión a altas temperaturas (en vehículos que utilizan diesel) se forman los $\mathrm{NO}_{\mathrm{x}}$.

La generación de $\mathrm{SO}_{\mathrm{x}}$ representa el $0.39 \%$ de las emisiones totales (699.6 ton/año), el transporte vehicular representa el $86.5 \%$ de este gas.

Existen diversas investigaciones como las de Bravo Ordoñez (2000) y CEPAL (2002), que plantean, entre otras estrategias el cambio de combustible que contenga menos azufre a fin de disminuir las emisiones de SOx; en efecto en Cusco, el 86.5\% del total de emisiones corresponde a la actividad vehicular y, ciertamente la desulforización sería una alternativa. Horc M. (2000) y EPA, 1991), sostienen que el ordenamiento del tránsito vehicular, podría disminuir en un $25 \%$ las emisiones en este rubro; en efecto, el trabajo del GESTA demuestra que más del $89 \%$ del total de emisiones corresponde a la actividad vehicular; por lo tanto, esta medida es adecuada.

Onursal B. (1997), afirma que un clima soleado contribuye a la formación de NOx en la atmósfera; la cuenca atmosférica del Cusco posee un clima altamente soleado con más de ocho horas sol en los meses de mayo a setiembre, por lo tanto, existe dicha posibilidad. Saavedra J.D. (2014) concluye que el parque automotor siempre será la fuente de mayor contaminación por gases y ruido; nuestro estudio, además, señala que también es fuente de contaminación por $\mathrm{PM}_{10}$ y polvo fugitivo.

Seinfeld J.H. (1986), OMS (2009) consideran que los contaminantes primarios emitidos por las fuentes fijas y móviles, son las que producen reacciones fotoquímicas agravando el problema en la salud pública; las observaciones de campo y la información proveniente del modelo MODEM, afirman que esto es una realidad también para Cusco

\section{CONCLUSIONES}

Las emisiones anuales generadas en la cuenca atmosférica del Cusco determinan como contaminante predominante al $\mathrm{PM}_{10}$ con una emisión total de 151,553.2 Ton/año; correspondiendo el $98.3 \%$ de esas emisiones al parque automotor. 
El monóxido de carbono constituye el segundo contaminante más abundante con 17,758.2 Ton/año. Los principales emisores son: el parque vehicular con el $38.6 \%$; las ladrilleras y tejerías con el $31.4 \%$ y las viviendas aportan con el $22.2 \%$ de las emisiones de CO.

Las emisiones de compuestos orgánicos volátiles (COV) alcanzan 6,308.2 Ton/año. Siendo los principales contribuyentes: Viviendas con el $31.6 \%$ de dichas emisiones; ladrilleras y tejerías con el $29.0 \%$ y el parque automotor con el $20.1 \%$.

Los gases $\mathrm{SO}_{2}$ y NOx tienen una presencia baja pero importante en las emisiones de la cuenca atmosférica, alcanzando 699.6 y 2,684.5 Ton/año respectivamente. El principal emisor de estos gases es el parque automotor, es el responsable del $86.5 \%$ de la emisión de SOx y del $90.8 \%$ de las emisiones de NOx respectivamente.

Para el caso del $\mathrm{SO}_{2}$, el ECA señala que, para un periodo de toma de muestra de 24 horas, el valor de referencia es $365 \mathrm{ug} / \mathrm{m}^{3}$, todos los valores muestreados están muy por debajo de este valor en todas las estaciones de muestreo.

Para las emisiones de $\mathrm{NO}_{x}$, el ECA reglamenta valores anuales y horarios. De los datos reportados se puede apreciar que en las estaciones de muestreo que tienen alta circulación vehicular se tienen valores altos, donde hay mayor posibilidad de dispersión por efecto de vientos; este contamínate no ha sobrepasado los valores de referencia.

El parque automotor es la fuente principal de contaminación en el Cusco; con el crecimiento económico el parque automotor se ha incrementado; por lo tanto, también la contaminación.

Los Valores de $\mathrm{NO}_{2}$ y $\mathrm{SO}_{2}$, indican que la contaminación viene de los taxis, combis, autos en mal estado y de carros rurales a diésel. Los valores de $\mathrm{NO}_{2}$ no son críticos, pero, este es un punto de partida para la formación de Ozono troposférico.

El ordenamiento del tránsito y tráfico vehicular, constituye una medida prioritaria para disminuir las emisiones procedentes del parque automotor, acción que corresponde a la Municipalidad provincial del Cusco.

\section{REFERENCIAS BIBLIOGRÁFICAS}

Anuario Estadístico de Hidrocarburos 2000. Ministerio de Energía y Minas. Dirección General de Hidrocarburos. Dirección de Promoción y Estadística. Lima.

Asociación de Gobernadores del Oeste Denver - Colorado y el Comité Asesor Binacional. Radian International. 1997. Manuales del Programa de Inventarios de Emisiones de México. Volumen III - Técnicas Básicas de Estimación de Emisiones. México.

Banco Interamericano de Desarrollo. 2013. Estrategias de mitigación y métodos para la estimación de emisiones en el sector transporte. BID-División de Transporte. Washington D.C., U.S.A

Blazer Martín. 2000. Dispersión de la contaminación atmosférica causada por tráfico vehicular, Swisscontact. Lima.

Bravo Ordoñez Adriana, Figari Bello Aida, Pareja Schroth. 2000. Emisiones de los vehículos automotores y planteamiento de posibles estrategias de control en el área metropolitana de Lima y Callao, Universidad Agraria de La Molina. Lima.

Calkins D. 1999. Revisión, análisis y Evaluación de los ECA propuestos para el Perú. Proyecto SENREM USAID/ Perú y CONAM, CONAM (Consejo Nacional del 
Ambiente). 1999. Programa Anual 1999 Para Estándares De Calidad Ambiental Y Límites Máximos Permisibles. En: www.conam.gob.pe

CEPAL (Comisión Económica para América Latina y el Caribe). 2002. Estudio económico de América Latina y el Caribe. "La congestión de tránsito: sus consecuencias económicas y sociales", Boletín N 170, Santiago de Chile.

Comisión Nacional del Medio Ambiente. 2009. Guía metodológica para la estimación de emisiones atmosféricas de fuentes fijas y móviles en el registro de emisiones y transparencia de contaminantes. Santiago. Chile.

D’Angiola, A.; Dawidowski, L.; Gómez, D.; Osses, M. (2010). On road traffic emissions in a megacity. Atmospheric Environment,44, 483-493

Decreto Supremo № 003-2017-MINAM. 2017. Estándares de Calidad Ambiental (ECA) para Aire y establecen Disposiciones Complementarias. El Peruano. Lima.

Decreto Supremo № 074-2001-PCM. 2001. Reglamento de estándares nacionales de calidad ambiental del aire. Lima.

De Nevers Noel, Ingeniería de Control de la Contaminación del Aire, McGraw Hill, USA, 1998.

DIGESA (2005). Resultados del inventario de emisiones de fuentes fijas cuenca atmosférica de la ciudad de Lima -Callao. Lima: Dirección General de Salud Ambiental.

Economopoulos, Alexander. (1993). Evaluación de Fuentes de Contaminación del Aire. Parte I: Técnicas para el Inventario Rápido de la Contaminación Ambiental. Organización Mundial de la Salud. Ginebra. 1993.

EPA (1997). United States. Environmental Protection Agency. Emission Inventory Improvement Program (EIIP), volume V. Biogenic sources. Preferred and alternatives methods. U.S. Environmental Protection Agency.

EPA (1999). United States. Environmental Protection Agency. Handbook for criteria pollutant inventory development: A beginner's guide for point and area sources. United States: Office of Air Quality Planning and Standards

Gil Mora, J.E. 2000. Cúpula de Aire Contaminado en la baja tropósfera del Cusco. Municipalidad Provincial del Cusco. Comisión Medio Ambiente, Regidores. Cusco.

Giraldo, L; Behrentz, E. 2009. Estimación del inventario de emisiones de fuentes móviles para la ciudad de Bogotá e identificación de variables pertinentes. MINAMBIENTE. Bogotá.

Instituto Nacional de Ecología y Cambio Climático-INECC, 2002. Tecnoteca, Tecnologías de control de contaminantes procedentes de fuentes estacionarias, formato pdf., Instituto Nacional de Ecología y Cambio Climático, México D.F.

IPIECA. (2019). IPIECA climate change reporting framework. Revisado en http://www.ipieca.org/resources/good-practice/ipieca-climate-change-reportingframework

Kageyama M., Sanin Aguirre L., Romieu I. 2001. Manual de Muestreo poblacional. Aplicaciones en Salud Ambiental. OMS, OPS, Centro panamericano de Ecología Humana. México.

Korc Marcelo, Mildred Maisonet. 2000. Directrices para la elaboración de los Planes de acción para el Mejoramiento de la Calidad del Aire, OPS/OMS-CEPIS. Lima.

Ministerio del Ambiente y Desarrollo Sostenible-MINAMBIENTE- Colombia. (2017). Guía para la elaboración de inventarios de emisiones atmosféricas. MINAMBIENTE. Bogotá.

OMS/EGH. 1997. Taller Sobre Contaminación Atmosférica Causada Por Vehículos Automotores. (Apéndice Del 1 Al 15). Banco Mundial Washington D.C. 
Onursal, B. Gautam S. P. 1997. Contaminación Atmosférica Por Vehículos Automotores, Experiencias Recogidas En Siete Centros Urbanos De América Latina. Documento Técnico del Banco Mundial Número 373S. Washington D.C.

Organización Panamericana de la Salud, 1998 Atención Primaria ambiental (APA). Washington D.C.

Osses Alvarado, Mauricio. 2000. Modelo de emisiones vehiculares, MODEM. Universidad de Chile. Santiago, Chile. $14 \mathrm{p}$.

Radian, 1996. Manuales del programa de inventarios de emisiones de México. Volumen IV - Desarrollo del inventario de fuentes puntuales. Radian International, Sacramento, CA

Robinson, E., and R.C. Robbins, 1968. Sources, Abundance, and Fate of Gaseous Atmospheric Pollutants. Report SRI Project PR6755, Stanford Research Institute, Menlo Park, California, pp. 1-122.

Saavedra Vargas, Juan Diego. 2014. Análisis de nuevos escenarios de emisión de contaminantes del parque automotor generados en un ambiente de tráfico vehicular. Tesis Ingeniero Ambiental. Universidad Agraria La Molima. Lima. 114 p.

Schipper, L., et al. (2009) "Considering Climate Change in Latin American and Caribbean Urban Transport: Concepts, Applications and Cases", Universidad de California. USA.

Seinfeld, J.H., 1986. Atmospheric Chemistry and Physics of Air Pollution. John Wiley \& Sons, Inc., New York.

SENAMHI-PACC. 2011. Caracterización climática delas regiones de Cusco y Apurimac. Ministerio del Ambiente. Lima. 131 p.

Secretaría de Medio Ambiente y recursos Naturales-SEMARNAT. 2012. Guía metodológica para la estimación de emisiones de fuentes fijas. talleres de Punto Final Gráficos S.A. de C.V. Tlalnepantla. México.

U.S. EPA, 1991. Procedures for Preparing Emissions Projections. U.S. Environmental Protection Agency, Office of Air Quality Planning and Standards, Research Triangle Park, North Carolina. EPA 450/4-91-019. Revisado en http://www.epa.gov/ttn/chief/ei guide.html.

WHO. 2009. Carbon Footprint Study FY2008- Final Report V2. Johannesburg, South Africa. $45 \mathrm{p}$.

WHO (2016). News release: World Health Assembly highlights importance of multisectoral action on health. Available online at: http://www.who.int/mediacentre/news/releases/2016/WHA69-importance-ofmultisectoral-action/

World Bank (2015). Connecting Climate Change and Health. World Bank Publications. Washington DC, USA 